\title{
Bendamustine-Bortezomib-Dexamethasone (BVD) in the management of relapsed and refractory Multiple Myeloma: a real-life experience
}

Cerchione C, Catalano L, Pareto AE, Basile S, Marano L, Peluso I, Simeone L, Vitagliano O, Romano A, Palmieri S, Rocco S, Pane F, Ferrara F

Division of Hematology, Department of Clinical Medicine and Surgery - University Federico II - Naples - Italy

Introduction

Bendamustine is a bifunctional alkylating agent, with low toxicity, proved to be effective in relapsed, refractory and in new diagnosed Multiple Myeloma (MM).

\section{Aims}

It has been evaluated efficacy and tolerance of Bendamustine, in combination with bortezomibdexametasone (BVD) in patients with relapsed and refractory MM (rrMM), whose prognosis is particularly severe. A regional retrospective reallife analysis of patients with rrMM who had been treated with BVD as salvage therapy has been performed.

Protocol schedule

Bendamustine $90 \mathrm{mg} / \mathrm{sqm}$ i.v. days 1,2

Bortezomib 1/1.3 mg/sqm s.c. days 1, 4, 8,11

Dexamethasone $20 \mathrm{mg}$, p.o., days 1, 2, 4, 5, 8, 9, 11, 12

Supportive care

Pegfilgrastim $6 \mathrm{mg}$ s.c. day +4

$>$ Levofloxacin $500 \mathrm{mg} / \mathrm{d}$, p.o., days 9-21 $>$ ESAs s.c. if required

Cycles were repeated every 28 days, until progression
Conclusion

Bendamustine-Bortezomib-Dexamethasone has shown significant efficacy in a particularly severe setting of patients, relapsed and refractory to all available therapeutic resources, and, in particular cases, it could be considered as a bridge to a second autologous or allogenic SCT.

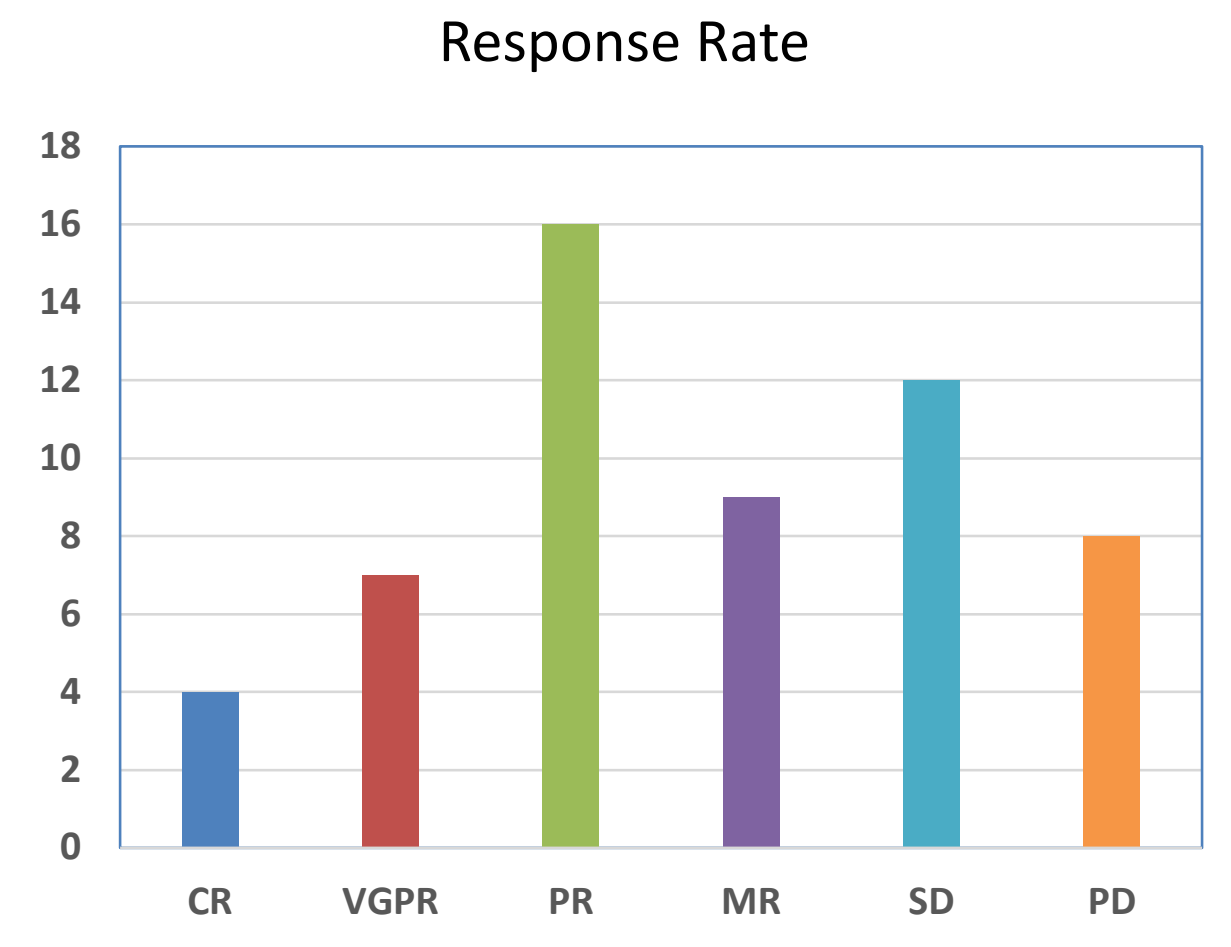

\begin{tabular}{|l|c|}
\hline Overall response rate - no. $(\%)(\geq \mathrm{MR})$ & $36 / 56(64 \%)$ \\
\hline Best response - no. (\%) & \\
\hline Stringent complete response (sCR) - no. (\%) & $1 / 56(1.8 \%)$ \\
\hline Complete response (CR) - no. (\%) & $3 / 56(5.3 \%)$ \\
\hline Very good partial response (VGPR) - no. (\%) & $7 / 56(12.5 \%)$ \\
\hline Partial response (PR) - no. (\%) & $16 / 56(28.5 \%)$ \\
\hline Minimal response (MR) - no. (\%) & $9 / 56(16 \%)$ \\
\hline Stable disease (SD) - no. (\%) & $12 / 56(21.4 \%)$ \\
\hline Progressive disease (PD) - no. (\%) & $8 / 56(14.3 \%)$ \\
\hline
\end{tabular}

\begin{tabular}{|c|c|c|c|c|c|c|c|c|c|}
\hline & Numero pazienti & Età diagnosi & Età inizio th & $\begin{array}{r}\text { Terapie } \\
\text { precedenti }\end{array}$ & ORR: ( $\geq P R$ ) & ORR: ( $\geq S D)$ & OS da diagnosi & $\begin{array}{r}\text { OS da inizio } \\
\text { th }\end{array}$ & TTR \\
\hline Pom-Dexa & $\begin{array}{c}22 \\
(13 \mathrm{M} / 9 \mathrm{~F})\end{array}$ & $\begin{array}{c}68 \\
\text { (r. 54-80) }\end{array}$ & $\begin{array}{c}71.5 \\
\text { (r. 55-83) }\end{array}$ & 5 (r. 2-8) & $40.9 \%$ & $77.2 \%$ & $\begin{array}{c}84 \\
\text { (r. 27-228) }\end{array}$ & $\begin{array}{c}8 \\
\text { (r. 1-14) }\end{array}$ & $\begin{array}{c}2 \\
(\text { r. } 1-4)\end{array}$ \\
\hline KRD & $\begin{array}{c}21 \\
(12 \mathrm{M} / 9 \mathrm{~F})\end{array}$ & $\begin{array}{c}62 \\
\text { (r. 47-75) }\end{array}$ & $\begin{array}{c}65 \\
\text { (r. 53-81) }\end{array}$ & 3 (r. 2-10) & $76.1 \%$ & $85.7 \%$ & $\begin{array}{c}47 \\
\text { (r. } 9-170)\end{array}$ & $\begin{array}{c}5 \\
\text { (r. 1-13) }\end{array}$ & $\begin{array}{c}2 \\
(\text { r. } 1-3)\end{array}$ \\
\hline VRD & $\begin{array}{c}22 \\
(16 \mathrm{M} / 6 \mathrm{~F})\end{array}$ & $\begin{array}{c}56 \\
\text { (r. } 38-74)\end{array}$ & $\begin{array}{c}59.5 \\
\text { (r. 54-69) }\end{array}$ & 2.5 (r. 1-5) & $50 \%$ & $77.2 \%$ & $\begin{array}{c}48 \\
\text { (r. 12-214) }\end{array}$ & $\begin{array}{c}26 \\
\text { (r. 15-39) }\end{array}$ & $\begin{array}{c}3 \\
(\text { r. } 1-3)\end{array}$ \\
\hline BVD & $\begin{array}{c}56 \\
(31 \mathrm{M} / 25 \mathrm{~F})\end{array}$ & $\begin{array}{c}57.3 \\
\text { (r. } 36-82)\end{array}$ & $\begin{array}{c}61.8 \\
\text { (r. } 37-83)\end{array}$ & 6 (r. 2-11) & $64 \%$ & $85.7 \%$ & $\begin{array}{c}62.7 \\
\text { (r. 6-151) }\end{array}$ & $\begin{array}{c}9.8 \\
\text { (r. } 2-36)\end{array}$ & $1.2(r .1-3)$ \\
\hline
\end{tabular}

